Mater. Res. Soc. Symp. Proc. Vol. 1611 (C) 2014 Materials Research Society

DOI: 10.1557/opl.2014.769

\title{
Cement Composites Reinforced with Functionalized Carbon Nanotubes
}

\author{
Sergey Petrunin ${ }^{1}$, Viktor Vaganov ${ }^{1}$, Konstantin Sobolev ${ }^{2}$ \\ ${ }^{1}$ Department of Nanotechnology and Theoretical Physics, Vladimir State University, Vladimir, \\ Russia \\ ${ }^{2}$ Department of Civil Engineering and Mechanics, University of Wisconsin-Milwaukee, \\ Milwaukee, USA
}

\begin{abstract}
This paper reports on the effect of surface functionalization of multi-wall carbon nanotubes on the strength and structure of portland cement composites. Reference nanotubes and nanotubes functionalized by carboxylic groups are used in this research.

Grafting of functional groups on the surface of the nanotubes allows the acceleration of cement hydration. It is established that the use of carboxylated nanotubes contributed to early strength development. The multi-wall carbon nanotubes reinforced composites are characterized by the high content of the calcium silicate hydrates and a very dense structure.
\end{abstract}

\section{INTRODUCTION}

The development of nanotechnology has enabled the creation of concrete with improved physical, mechanical and durability characteristics $[1,2]$. Carbon nanotubes (CNTs) have very unique properties: a very high Young's modulus of up to $630 \mathrm{GPa}$, strength up to $150 \mathrm{GPa}$, high electrical and thermal conductivity. Reinforcing the cement matrix by CNTs allows the development of material with enhanced performance characteristics [1-3].

In this paper, the effect of surface functionalization of multi-wall carbon nanotubes (MWCNTs) on strength, structure and composition of portland cement composites are investigated. Reference nanotubes and nanotubes functionalized by carboxylic groups are compared in the research.

Cement composites modified by CNTs have improved strength, corrosion and freeze-thaw resistance in comparison with the reference [4-6]. However, the effective use of CNTs as a reinforcing material in concrete is complicated, because CNTs are chemically inert and, under the influence of Van der Waals forces, have a tendency to form agglomerates [7, 8]. Grafting of oxygen-containing groups on the surface of CNTs enables chemical interaction between the nanotubes and the cement matrix $[9,10]$. It is established that oxygenized CNTs are involved in the hydration process of cement and form the bonds with the cement hydration products [11]. The interaction of carboxylated CNTs with cement hydration products is investigated. In this case, the adsorption of hydrates on surfaces of CNT is observed and resulted in a higher strength vs. concrete modified with reference CNTs. 


\section{THEORY}

\section{Materials}

Ordinary portland cement CEM I $42.5 \mathrm{R}$ is used for the synthesis of CNT-reinforced composite. Silica sand with a fineness modulus of 2.8 is used as a fine aggregate. Polycarboxylate superplasticizer is used to enhance the stability of the CNT colloidal solution in water and reduce the agglomeration effects inherent to nano-objects. The MWCNTs are used as a reinforcing component for the composite. The initial MWCNTs are in the form of filaments with a diameter of 30-60 nm bound and rolled up into the balls. Fig. 1 shows the SEM and TEM images of carbon nanotubes after synthesis and carboxylation.
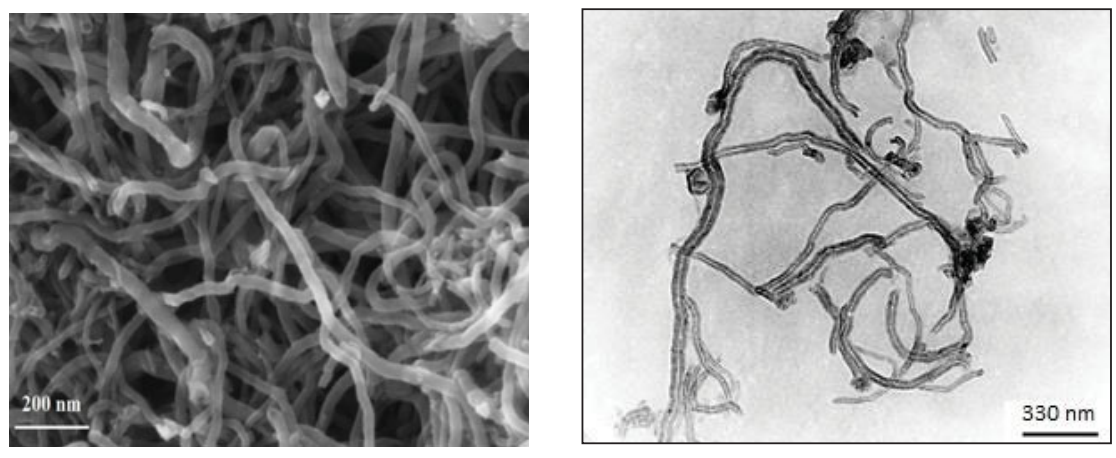

Figure 1. The SEM image (left) and TEM (right) of carboxylated MWCNTs

\section{Preparation of Specimens}

All composites are produced at a sand to cement ratio of 1 and water to cement ratio $(\mathrm{w} / \mathrm{c})$ of 0.3 . The concentration of carbon nanotubes ranged from $0.01 \%$ to $0.25 \%$ by weight of cement. The carbon nanotubes are introduced into a solution of water and a polycarboxylate plasticizer with the concentration of MWCNTs varying from $0.75 \%$ to $6 \%$. This dispersion is sonicated at $20 \mathrm{kHz}$ using the ultrasound processor (IL100-6/1-1) at maximal power (700 W) for 15 minutes. Cement and sand are added into a planetary mixer and mixed for 3 minutes. Then the required amount of water with polycarboxylate superplasticizer and carbon nanotubes is added into the mixer. The entire mixture is stirred for 7 minutes (Fig. 2). After this procedure, the composite mixture is placed into the metal molds with dimensions of $40 \times 40 \times 160 \mathrm{~mm}$ and compacted by vibration. The composite specimens are demolded after 24 hours of initial hardening and are stored in lime water until the testing age. 


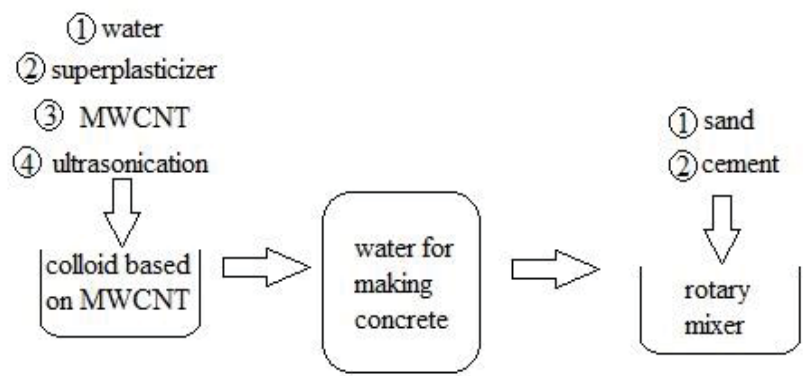

Figure 2. Preparation of MWCNT cement based composite

\section{Functionalization of MWCNT by Carboxylic Groups}

MWCNTs are added to the flask and filled with a mixture of mineral acids at a rate of 1:5. The mixture is stirred at $70^{\circ} \mathrm{C}$ for 24 hours. Then the MWCNT nanomaterial is filtered, washed with water, ethanol and dried.

\section{Testing Procedure}

The morphology and microstructure of the CNT nanomaterial and suspensions are studied by Scanning Election Microscope (SEM) Zeiss LEO SUPRA 25 and Transmission Electron Microscope (TEM) JEM 200 LV. The composite specimens are tested for compressive strength at the age of 1,7 and 28 days.

\section{DISCUSSION}

\section{Mechanical properties}

The dispersion of carbon nanotubes functionalized by carboxyl groups is studied using TEM. Fig. 1 shows a micrograph of dispersion of carboxylated MWCNTs. It can be observed that MWCNTs are well separated and have a diameter of 30-60 nm and a length from $100 \mathrm{~nm}$ to several microns.

The compressive strength test results of composites with different content of reference MWCNTs at the age of 1,7 and 28 days are given in Fig. 3. The compressive strength improvement of $37 \%$ and $20 \%$ after 1 day and 28 days of curing, respectively, are observed for "pure" unmodified MWCNT composites. The composite with MWCNT dosage of $0.13 \%$ (by the weight of cement) has a maximal compressive strength of $64 \mathrm{MPa}$.

Fig 4 represents the results of compressive strength of cement composites modified with $0.05 \%$ (by the weight of cement) of carboxylated MWCNT's. It is observed that the use of carboxylated CNTs accelerates the hydration of portland cement and results in $30 \%$ increase of 
1-day compressive strength; however, the composites reinforced by the "pure" unmodified CNTs had a better performance in 28-day age (Fig. 4).

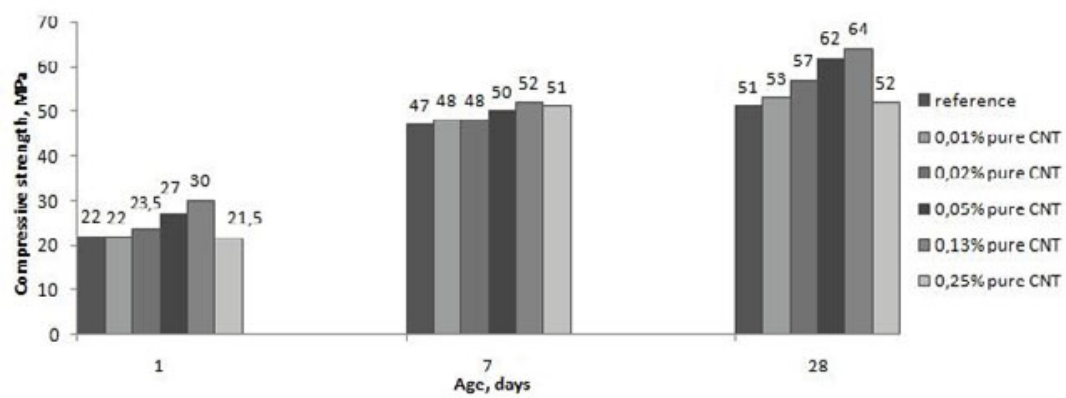

Figure 3. The Compressive Strength of Composites with Reference MWCNT (by the weight of cement)

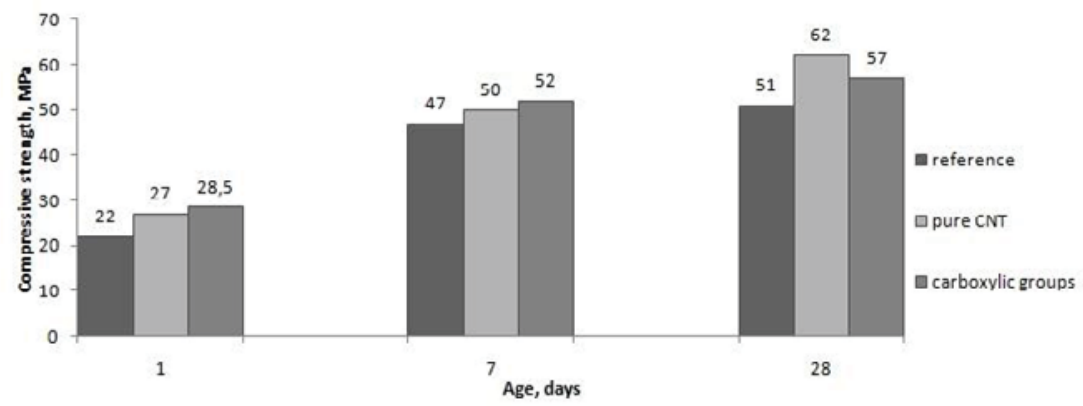

Figure 4. The Compressive Strength of Composites with 0.05\% Carboxilated CNTs (by the weight of cement)

In the process of cement hydration the $\mathrm{OH}^{-}, \mathrm{Ca}^{2+}, \mathrm{CaOH}^{-}$and $\mathrm{H}_{2} \mathrm{SiO}_{4}{ }^{2-}$ ions are deposited on the surface of cement grains and form the layer consisting of C-S-H gel with embedded $\mathrm{Ca}(\mathrm{OH})_{2}$ crystals (Fig. 5a). With time the thickness of layer increases and its ionic permeability decreases. The formation of C-S-H gel and $\mathrm{Ca}(\mathrm{OH})$ takes place when $\mathrm{Ca}^{2+}$ concentration exceeds the saturation limit by factor of 1.5-2. The intensification of hydration in the system with CNTs is due to an additional sorption of $\mathrm{Ca}^{2+}$ ions on the surface of CNTs. In this case, the hydration is accelerated, since the formation of C-S-H and $\mathrm{Ca}(\mathrm{OH})_{2}$ crystals in the areas supersaturated by $\mathrm{Ca}^{2+}$ ions. Fragmentary formation of hydration products on CNTs surface contributes to the improvement of ion exchange between the solid and liquid phases and results in an acceleration of formation of new phases (Fig. 5b). The presence of oxygen-containing groups on the surface of CNTs leads to a more active sorption $\mathrm{Ca}^{2+}$ ions and higher rates of hardening in case when functionalized CNTs are used (Fig. 5c). 

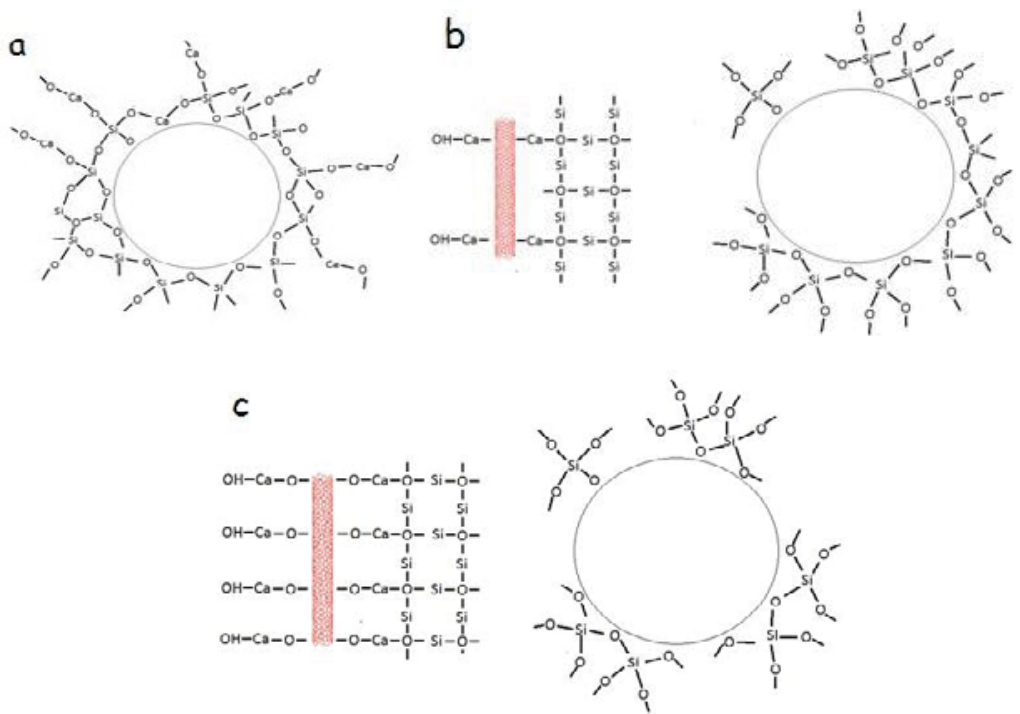

Figure 5. The schematic of hydration process: a) without CNT, b) in the presence of pure CNT, c) in the presence of carboxylated CNTs

\section{SEM analysis}

Figure 6 displays the micrograph of cement composite structure modified by carboxylated CNTs. The micropockets filled with filamentary nanostructured formations, based on carbon nanotubes can be observed. These formations are surrounded by the hydration products forming three-dimensional network and binding neighboring clusters of C-S-H gel. With the addition of carboxylated CNTs the size of micropores decreases resulting in a stronger cementitious matrix which improves the performance of composite.
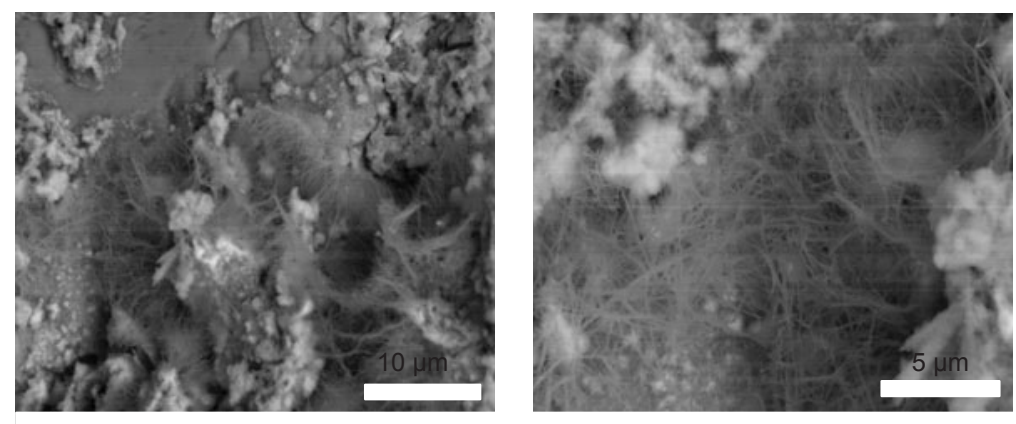

Figure 6. The Microstructure of Cement Composite Modified with Carboxylated CNTs 


\section{CONCLUSIONS}

The effect of dosage and surface functionalization of multi-walled carbon nanotubes on the strength of cementitious composites is investigated. The maximal compressive strength of 64 $\mathrm{MPa}(20 \%$ increase over the reference) is observed in the composite with $0.13 \%$ (by the weight of cement) of MWCNTs. It is established that grafting of carboxylate functional groups on the surface of the nanotubes accelerates the hydration of portland cement and improves early strength. The addition of carboxylated MWCNTs at a very low dosage of $0.05 \%$ provides a $30 \%$ increase of 1-day compressive strength of MWCNT-composites.

\section{ACKNOWLEDGMENTS}

The authors acknowledge the financial support from Bortnick foundation under the program «UMNIK» (Grant 2012). KS is thankful for the financial support provided by CFIRE and UWMRGI.

\section{REFERENCES}

1. K. Sobolev and M. Ferrada-Gutiérrez, Am. Ceram. Soc. Bull. 10, 14-17 (2005).

2. F. Sanchez and K. Sobolev, Constr. Build. Mater. 24, 2060-2071 (2010).

3. M.S. Konsta-Gdoutos, Z.S. Metaxa, S.P. Shah, Cement Concrete Res. 40, 1052 (2010).

4. L. I. Nasibulina, I. V. Anoshkin, A. V. Semencha, O. V. Tolochko, J. E. M. Malm, M. J. Karppinen, A. G. Nasibulin, E. I. Kauppinen, Mater. Phys. Mech. 13, 77 (2012).

5. G.I.Yakovlev, G.N. Pervushin, A. Korzhenko, A.F. Buryanov, I.A. Pudov, A.A. Lushnikova, Constr. Mater. 2, 47 (2011), in Russian.

6. S.Y. Petrunin, M.Y. Popov, V.E. Vaganov, V.V. Reshetniak, L.V. Zakrevskaya, Nanotech. Constr. 5, 65 (2012), in Russian.

7. E.G. Rakov, Russ. Chem. Rev. 70, 827 (2001).

8. A. Sobolkina, V. Mechtcherine, V. Khavrus, D. Maier, M. Mende, M. Ritschel, A. Leonhardt, Cement Concrete Comp. 34, 1104 (2012).

9. G.Y. Li, Pei Ming Wang, Xiaohua Zhao, Carbon 43, 1239 (2005).

10. G. Yakovlev, J. Keriene, A. Gailius, I. Girniene, J. Mater. Sci. 12, 147 (2006).

11. L. I. Nasibulina, I.V. Anoshkin, A. G. Nasibulin, A. Cwirzen, V. Penttala, E. I. Kauppinen, J. Nano Mater. 1, 6 (2012). 